\title{
Estimation of Dispersion Parameters
}

\author{
W. A. Thompson, Jr. ${ }^{1}$
}

(July 31, 1962)

\begin{abstract}
This paper deals with a topic in multivariate analysis. Consider that a sample of size $n+1$ has been collected from a $p$-variate normal distribution having dispersion matrix $\left(\sigma_{i j^{\prime}}\right)$. Let $a_{i j^{\prime}} / n$ denote the usual unbiased estimate of $\sigma_{i j^{\prime}}$. Further, let $0<l<u$ be constants such that all characteristic roots of a matrix having the Wishart distribution lie in the interval $[l, u]$ with probability $1-\alpha$. A theorem of Roy, Bose, and Gnanadesikan [Ann. Math. Stat. 24, 513-536 (1953); Biometrika 44, 399-410 (1957)] may be stated as follows: The probability is $1-\alpha$ that every principal minor determinant of $l^{-1}\left(a_{i j^{\prime}}\right)-\left(\sigma_{i j^{\prime}}\right)$ and of $\left(\sigma_{i j^{\prime}}\right)-u^{-1}\left(a_{j j^{\prime}}\right)$ is nonnegative. The previous result may be used to prove the main theorem of the present paper. Theorem: The probability is at least $1-\alpha$ that the following system of relations hold simultaneously: $u^{-1} a_{j j} \leq \sigma_{i j} \leq l^{-1} a_{j i} ; j=1, \ldots, p$ and $\mid \sigma_{j j^{\prime}}-1 / 2$ $\left(u^{-1}+l^{-1}\right) a_{j j^{\prime}} \mid \leq 1 / 2\left(l^{-1}-u^{-1}\right)\left(a_{j j} a_{j^{\prime} j^{\prime}}\right)^{1} / 2, j \neq j^{\prime}$.
\end{abstract}

\section{Notation}

'Throughout $Y_{i}=\left(y_{i 1}, \ldots ., y_{i p}\right)$ ' for $i=1$, . ., $N$ will be a sample of size $\hat{N}_{=}=n+1$ from a $p$-variate normal distribution with mean vector $\xi=\left(\xi_{1} \ldots \xi_{p}\right)^{\prime}$ and dispersion matrix $\Sigma$. In short, the $Y_{i}$ are independent and $N(\xi, \Sigma)$. Except for $\alpha$ and $\sum$, Greek letters will always refer to parameters. We will frequently write $\Sigma=\left(\sigma_{j j^{\prime}}\right)$ when we mean that $\sigma_{j j^{\prime}}$ is the element in the $j$ th row and $j^{\prime}$ th column of $\Sigma$. In the same spirit $\Sigma^{-1}=\left(\sigma^{j j^{\prime}}\right)$, and $A=\left(a_{j j^{\prime}}\right)$ will be common notations. Here

$$
a_{j j^{\prime}}=\sum_{i=1}^{N}\left(y_{i j}-\bar{y}_{i j}\right)\left(y_{i j^{\prime}}-\bar{y}_{i j^{\prime}}\right) .
$$

\section{Simultaneous Confidence Intervals}

Let $0<l<u$ be constants such that ail characteristic roots of a $p \times p$ matrix having the distribution $W(I, n)$ (see $[1]^{2}$ for this notation) are in the interval $[l, u]$ with probability $1-\alpha$. The following theorem is, for our purposes, a more convenient statement of some results due to Roy, Bose, and Gnandesikan [8, 9].

Theorem 1. The probability is $1-\alpha$ that $l^{-1} A-\Sigma$ and $\Sigma-u^{-1} A$ are positive semi-definite.

A proof is included since the original verification demonstrates many other interesting results as well, and consequently is very indirect. There exists a nonsingular triangular matrix $C$ such that $C \Sigma C^{\prime}=I$ [1, p. 156]. Making the transformation $Z_{i}=C Y_{i}(i=$ $1, \ldots, N)$ then $Z_{i}$ has the distribution $N(C \xi, I)$ and $B \equiv \sum_{i=1}^{N}\left(Z_{i}-\bar{Z}\right)\left(Z_{i}-\bar{Z}\right)^{\prime}=C A C^{\prime}$ has the distri-

1 On leave from the University of Delaware; National Academy of Sciences National Research Council Postdoctoral Resident Research Associate at the National Research Council Postdoctoral Resident Rese
National Bureau of Standards, academic year 1961-62.

2 Figures in brackets indicate the literature references at the end of this paper. bution $W(I, n)$. The theorem will be proved when it is shown that the following three statements are equivalent. (i) all roots of $B$ are in the interval $[l, u]$. (ii) $B-l I$ and $u I-B$ are p.s.d. (positive semidefinite). (iii) $A-l \Sigma$ and $u \Sigma-A$ are p.s.d. (i) and (ii) are clearly equivalent. That $B-l I$ and $A-l \Sigma$ are p.s.d. together may be shown by considering their quadratic forms. Making the transformation $Q=C R$, where $Q$ and $R$ are column vectors, we have $Q^{\prime}(A-l \Sigma) Q=R^{\prime}\left(C^{\prime} A C-l C^{\prime} \Sigma C\right) R=R^{\prime}(B-l I) R$. Similarly the matrices $u I-B$ and $u \Sigma-A$ are p.s.d. together.

An equivalent statement of theorem 1 is

Theorem $1^{\prime}$. The probability is $1-\alpha$ that every principal minor determinant of $l^{-1} A-\Sigma$ and of $\Sigma-u^{-1} A$ is nonnegative.

Proof. The event whose probability is being calculated in $1^{\prime}$ is identical with that of theorem 1 [3, theorem 46.4].

Clearly the previous theorem provides the means of determining a simultaneous confidence region for the elements of the dispersion matrix $\Sigma$. However this region, call it $\mathscr{R}$, may or may not be interesting depending on its shape. We now begin an investigation of the shape of $\mathscr{R}$. Let $\mathscr{P}=\left(\Sigma: l^{-1} A-\Sigma\right.$ is p.s.d.) and $\mathscr{T}=\left(\Sigma: \Sigma-u^{-1} A\right.$ is p.s.d. $)$. Then $\mathscr{R}=$ $\mathscr{P} \cap \mathscr{T}$, the intersection or common part of $\mathscr{S}$ and $\mathscr{T}$.

Theorem 2. $\mathscr{S}$ and $\mathscr{T}$ are convex cones with vertices $\Sigma=l^{-1} A$ and $\Sigma=u^{-1} A$, respectively.

Proof. Assume $\Sigma_{1} \epsilon \mathscr{S}, \quad \Sigma_{2} \epsilon \mathscr{S}$ and $c \geq 0$. Clearly $l^{-1} A \epsilon \mathscr{S}$. Further $c \Sigma_{1}+(1-c) l^{-1} A \epsilon \mathscr{S}$ since $l^{-1} A-$ $\left[c \Sigma_{1}+(1-c) l^{-1} A\right]=c\left(l^{-1} A-\Sigma_{1}\right)$. Hence $\mathscr{S}$ is a cone with vertex $l^{-1} A$. To show $\mathscr{S}$ is convex we must demonstrate that $c \Sigma_{1}+(1-c) \Sigma_{2} \epsilon \mathscr{S}$ whenever $c \leq 1$ But the quadratic form $Q^{\prime}\left[l^{-1} A-c \Sigma_{1}-(1-c) \Sigma_{2}\right] \bar{Q}=$ $c Q^{\prime}\left(l^{-1} A-\Sigma_{1}\right) Q+(1-c) Q^{\prime}\left(l^{-1} A-\Sigma_{2}\right) Q$ is nonnega tive and $\mathscr{S}$ is a convex cone. The proof for $\mathscr{T}$ is similar. 
According to theorem $2, \mathscr{R}$ is the intersection of two convex cones. Further on in the paper (theorem $3)$ we shall prove a result which implies that $\mathscr{R}$ is bounded. Hence the confidence region $\mathscr{R}$ is convex and bounded.

Now, in the case $p=2$, define

$$
\begin{gathered}
w_{1}=\sigma_{11}-u^{-1} a_{11}, w_{2}=\sigma_{12}-u^{-1} a_{12}, w_{3}=\sigma_{22}-u^{-1} a_{22}, \\
d_{1}=\left(l^{-1}-u^{-1}\right) a_{11}, d_{2}=\left(l^{-1}-u^{-1}\right) a_{12},
\end{gathered}
$$

$d_{3}=\left(l^{-1}-u^{-1}\right) a_{22}$,

$W=\left(\begin{array}{ll}w_{1} & w_{2} \\ w_{2} & w_{3}\end{array}\right)$ and $D=\left(\begin{array}{ll}d_{1} & d_{2} \\ d_{2} & d_{3}\end{array}\right)$.

$\mathscr{T}$ and $\mathscr{S}$ become $\left[w_{1}, w_{2}, w_{3}: w_{1} \geq 0, w_{3} \geq 0\right.$ and $w_{2}^{2} \leq$ $\left.w_{1} w_{3}\right]$ and $\left[w_{1}, w_{2}, w_{3}: w_{1} \leq d_{1}, w_{3} \leq d_{3}\right.$ and $\left(w_{2}-d_{2}\right)^{2} \leq$ $\left.\left(w_{1}-d_{1}\right)\left(w_{3}-d_{3}\right)\right]$, respectively. Solving simultaneously we find that the intersection of the boundaries of $\mathscr{S}$ and $\mathscr{T}$ takes place on the plane $d_{1} w_{3}+d_{3} w_{1}-2 d_{2} w_{2}=d_{1} d_{3}-d_{2}^{2}=|D|$. On this plane the equation of the required intersection is $d_{3} w_{2}^{2}=(|D|+$ $\left.2 d_{2} w_{2}-d_{1} w_{3}\right) w_{3}$ or

$$
d_{3} w_{2}^{2}-2 d_{2} w_{2} w_{3}+d_{1} w_{3}^{2}-|D| w_{3}=0
$$

which will be an ellipse since $D=\left(l^{-1}-u^{-1}\right) A$ is positive definite with probability one.

Since the vertices of $\mathscr{S}$ and $\mathscr{T}$ satisfy $(2.2)$ the extent of $w_{2}$ and $w_{3}$ in $\mathscr{R}$ will be the same as their extent in the ellipse. To find the extent of $w_{2}$ in (2.2) consider $w_{2}$ to be fixed and solve for $w_{3}$. (2.2) is then a quadratic equation with discriminant $\left(|D|+2 d_{2} w_{2}\right)^{2}-4 d_{1} d_{3} w_{2}^{2}$. If $w_{3}$ is to be real, then the discriminant must be nonnegative; hence the extent of $w_{2}$ is given by the relation $\left(\mid D+2 d_{2} w_{2}\right)^{2} \geq 4 d_{1} d_{3} w_{2}^{2}$ or equivalently $\left|w_{2}-d_{2} / 2\right| \leq 1 / 2\left(d_{1} d_{3}\right)^{1 / 2}$. The extent of $w_{3}$ is even easier to calculate by the same methods. Solving (2.2) for $w_{2}$ the discriminant reduces to $4|D| w_{3}\left(d_{3}-w_{3}\right)$, which yields $0 \leq w_{3} \leq d_{3}$. Similarly $0 \leq w_{1} \leq d_{1}$. Using theorem 1' and the eq (2.1) we summarize these computations as

Lemma 1 . In the case of $p=2$, the probability is at least $1-\alpha$ that the following three relations hold simultaneously

$$
\begin{aligned}
& u^{-1} a_{11} \leq \sigma_{11} \leq l^{-1} a_{11}, u^{-1} a_{22} \leq \sigma_{22} \leq l^{-1} a_{22} \\
& \quad\left|\sigma_{12}-\frac{u^{-1}+l^{-1}}{2} a_{12}\right| \leq \frac{l^{-1}-u^{-1}}{2}\left(a_{11} a_{22}\right)^{\frac{1}{2}} .
\end{aligned}
$$

Now consider the general case, where $p$ is not necessarily equal to 2 . In theorem $1^{\prime}$ we may choose to ignore all principal minor determinants of order greater than 2. If we do this, then the probability of the resulting event can only be increased. For each pair of variates, and by identical methods, we will obtain a system of relations just like those of lemma 1.

Theorem 3. The probability is at least $1-\alpha$ that the following system of relations hold simultaneously

$$
u^{-1} a_{j j} \leq \sigma_{j j} \leq l^{-1} a_{j j}, j=1, \ldots, p
$$

and

$$
\left|\sigma_{j j^{\prime}}-\frac{u^{-1}+l^{-1}}{2} a_{j j^{\prime}}\right| \leq \frac{l^{-1}-u^{-1}}{2}\left(a_{j j} a_{j^{\prime} j^{\prime}}\right)^{\frac{1}{2}}, j \neq j^{\prime} .
$$

\section{Precision of Instruments}

A special multivariate model which frequently arises in connection with simultaneous measurement procedures $[5,11]$ requires that $\sigma_{j j}=\sigma^{2}+\sigma_{j}^{2}, j=1$, . . . $p$ and $\sigma_{j j^{\prime}}=\sigma^{2}, j \neq j^{\prime}$. For this application, $p$ becomes the number of instruments used. The methods of the previous section yield interesting results when applied to this special case. Returning to eq $(2.2)$ we calculate the extent of $t=w_{3}-w_{2}$; this will yield bounds for $\sigma_{2}^{2}$ in the two instrument case. Making the substitution $w_{3}=t+w_{2}$, eq (2.2) becomes

$$
w_{2}^{2}\left(d_{1}+d_{3}-2 d_{2}\right)+2 t w_{2}\left(d_{1}-d_{2}\right)+d_{1} t^{2}-|D|\left(t+w_{2}\right)=0 .
$$

Computations similar to those made for $w_{2}$ show that the extent of $t$ is $\frac{1}{2}\left(d_{3}-d_{2}\right) \pm \frac{1}{2}\left[d_{3}\left(d_{1}+d_{3}-2 d_{2}\right)\right]^{\frac{1}{2}}$.

Equations (2.1) and theorem 1' now yield

Lemma 2. In the two-instrument case, the probability is at least $1-\alpha$ that the following three relations hold simultaneously

$$
\begin{aligned}
& \left|\sigma^{2}-a_{12} \frac{l^{-1}+u^{-1}}{2}\right| \leq \frac{l^{-1}-u^{-1}}{2}\left(a_{11} a_{22}\right)^{\frac{1}{2}}, \\
& \left|\sigma_{1}^{2}-\left(a_{11}-a_{12}\right) \frac{l^{-1}+u^{-1}}{2}\right| \leq \frac{l^{-1}-u^{-1}}{2}\left[a_{11}\left(a_{11}+a_{22}-2 a_{12}\right)\right]^{\frac{1}{2}}, \\
& \left|\sigma_{2}^{2}-\left(a_{22}-a_{12}\right) \frac{l^{-1}+u^{-1}}{2}\right| \leq \frac{l^{-1}-u^{-1}}{2}\left[a_{22}\left(a_{11}+a_{22}-2 a_{12}\right)\right]^{\frac{1}{2}} .
\end{aligned}
$$

For more than two instruments we may again choose to ignore all principal minor determinants of order in excess of 2 . 'Theorem $1^{\prime}$ then gives

Theorem 4. The probability is at least $1-\alpha$ that the following relations hold simultaneously

$$
\begin{array}{r}
\max _{j \neq j^{\prime}}\left[a_{j j^{\prime}} \frac{l^{-1}+u^{-1}}{2}-\frac{l^{-1}-u^{-1}}{2}\left(a_{j j} a_{j^{\prime} j^{\prime}}\right)^{\frac{1}{2}}\right] \\
\leq \sigma^{2} \leq \min _{j \neq j^{\prime}}\left[a_{j j^{\prime}} \frac{l^{-1}+u^{-1}}{2}+\frac{l^{-1}-u^{-1}}{2}\left(a_{j j} a_{j^{\prime} j^{\prime}}\right)^{\frac{1}{2}}\right] \\
\max _{j \neq 1}\left\{\left(a_{11}-a_{1 j_{j}}\right) \frac{l^{-1}+u^{-1}}{2}\right. \\
\left.\quad-\frac{l^{-1}-u^{-1}}{2}\left[a_{11}\left(a_{11}+a_{j j}-2 a_{1_{j}}\right)\right]^{\frac{1}{3}}\right\} \\
\leq \sigma_{1}^{2} \leq \min _{j \neq 1}\left\{\left(a_{11}-a_{1 j}\right) \frac{l^{-1}+u^{-1}}{2}\right. \\
\left.\quad+\frac{l^{-1}-u^{-1}}{2}\left[a_{11}\left(a_{11}+a_{j j}-2 a_{1_{j}}\right)\right]^{\frac{1}{2}}\right\} .
\end{array}
$$

plus similar inequalities involving $\sigma_{2}^{2}, \ldots . \sigma_{n}^{2}$. 


\section{Choosing the Bounds}

The joint distribution of the characteristic roots of a Wishart matrix is well known, see for example [1, theorem 13.3.2]. Also, there is much theoretical work on the distributions of the extreme roots [10]. Tables and charts have been prepared by Pillai [7] and Heck [6] from which critical values of the largest root may be determined for certain combinations of $n, p$, and $\alpha$. However, critical values of the largest root are of no value in this application without some knowledge of the smallest root. The tabulations of Pillai and Heck do not treat large values of their parameter $m$ and consequently, even though there is a relation between the distribution of the largest and smallest root, their results cannot be used to determine critical values of the smallest root in the present instance. The only result which seems to be ready for use in determining the bounds is an approximation due to P. L. Hsu (for an exposition see [2]). In our terminology, Hsu's result states that $P\left(l \leq \chi_{n-p+2}^{2}\right)$ is, for large $n$, an approximation to the probability that the minimum root is at least as large as $l$.

Here we present an exact treatment of the two variate case $(p=2)$. Denote the roots by $r$ and $s$ where $r \leq s$. Our task is to determine constants $l$ and $u$ such that $P(l \leq r \leq s \leq u)=1-\alpha$.

$$
\begin{aligned}
P & =P(l \leq r \leq s \leq u) \\
& =c \int_{s=l}^{u} \int_{r=l}^{s}(r s)^{\frac{n-3}{2}} \exp \left[-\frac{r+s}{2}\right](s-r) d r d s,
\end{aligned}
$$

where $c=\sqrt{\pi}\left[2^{n} \Gamma\left(\frac{n}{2}\right) \Gamma\left(\frac{n-1}{2}\right)\right]^{-1}$. Making the

transformation $r=t-v, s=t+v$ then $P / 4 c$ may be written as

$$
\begin{aligned}
\int_{t=l}^{\frac{l+u}{2}} e^{-t} \int_{v=0}^{t-l} v\left(t^{2}-v^{2}\right)^{\frac{n-3}{2}} d v d t & \\
& +\int_{t=\frac{l+u}{2}}^{u} e^{-t} \int_{v=0}^{u-t} v\left(t^{2}-v^{2}\right)^{\frac{n-3}{2}} d v d t .
\end{aligned}
$$

Now, integrating with respect to $v$ and transforming the resultant expressions, we obtain $(n-1) P / 2 c$ in the form

$$
\begin{aligned}
\int_{2 l}^{2 u}(x / 2)^{n-1} e^{-x / 2} d x & \\
& -\left(l^{\frac{n-1}{2}} e^{-\frac{l}{2}}+u^{\frac{n-1}{2}} e^{-\frac{u}{2}}\right) \int_{l}^{u} x^{\frac{n-1}{2}} e^{-\frac{x}{2}} d x .
\end{aligned}
$$

Finally remembering the value of $c$ and using the expression $\sqrt{\pi} \Gamma(n)=2^{n-1} \Gamma\left(\frac{n}{2}\right) \Gamma\left(\frac{n+1}{?}\right)$ we obtain

$$
\begin{aligned}
& P(l \leq r \leq s \leq u) \\
& =P\left(2 l \leq \chi_{2 n}^{2} \leq 2 u\right) \\
& -\frac{\sqrt{\pi}}{2^{\frac{n-1}{2}} \Gamma\left(\frac{n}{2}\right)}\left(l^{\frac{n-1}{2}} e^{-\frac{l}{2}}+u^{\frac{n-1}{2}} e^{\left.-\frac{u}{2}\right) P\left(l \leq \chi_{n+1}^{2} \leq u\right),}\right.
\end{aligned}
$$

where $\chi_{2 n}^{2}$ is a chi-square variate with $2 n$ degrees of freedom.

As is usual in such problems, there is considerable freedom in choosing $l$ and $u$ so that $P=1-\alpha$. In table $1, u$ is taken to be $+\infty$ and in table $2, l$ equals 0 . The bounds of table 3 are chosen as follows: Determine $l$ so that $P(l \leq r)=1-\alpha$, then holding $l$ fixed at this value determine $u_{2}$ so that $P\left(l \leq r \leq s \leq u_{2}\right)=1-2 \alpha$. Note that the value of $u_{2}$ obtained in this way agrees very closely with the corresponding entry of table 2 . It appears that the probabilistic dependence of the greatest and least root may be ignored for the purpose of this paper.

Table 1 was used to check the accuracy of Hsu's approximation in the bivariate case. The approximation runs from about 15 percent too large for $n=60$ to about 11 percent too large for $n=100$. Presumably the approximation would be equally poor in general, except for very large sample sizes.

TABLE 1. Percentage points 1 of the smallest characteristic root of a bivariate Wishart matrix having sample size $\mathrm{n}+1$

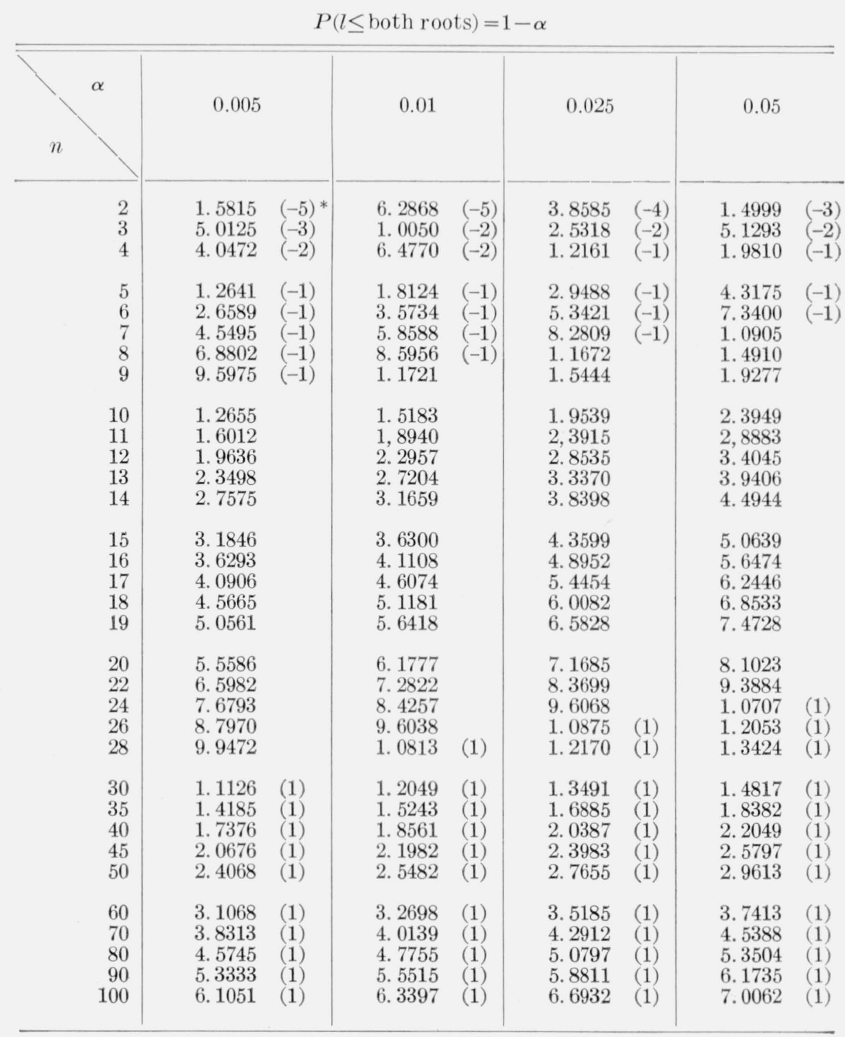

*The symbol (i) means that the tabled entry is to be multiplied by $10^{i}$. 
$\mathrm{T}_{\mathrm{ABLE}} 2$. Percentage points $\mathrm{u}_{1}$ of the largest characteristic root of a bivariate Wishart matrix having sample size $\mathrm{n}+1$

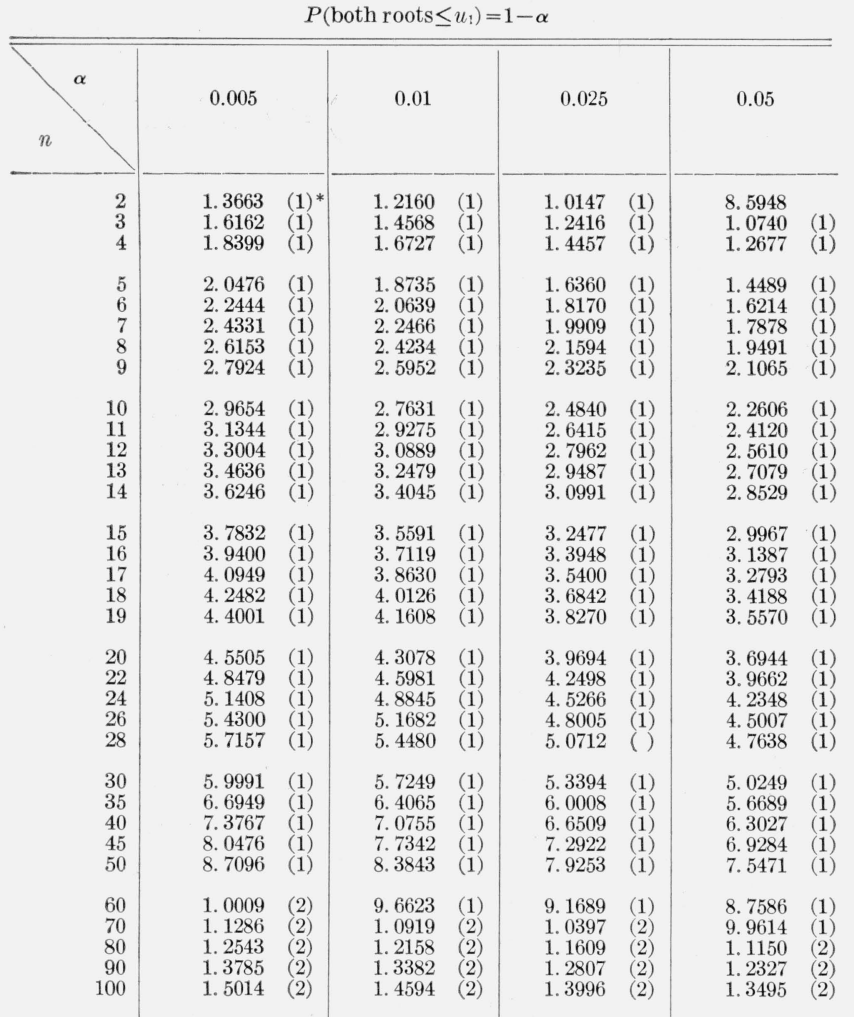

*The symbol $(i)$ means that the tabled entry is to be multiplied by $10^{i}$.

Following a verbal presentation of the results of this paper, certain unpublished portions of $\mathrm{R}$. Gnanadesikan's 1956 thesis [4] were kindly pointed out as being pertinent. Gnanadesikan has obtained general recursion formulae for calculating two-sided bounds on the characteristic roots of a Wishart matrix. In the bivariate case I have been able to verify, by partial integration that (4.1) is equivalent to Gnanadesikan's expression. Confidence intervals for the dispersion parameters were also obtained by Gnanadesikan. His result is different from that of theorem 3 and may provide an alternative approach to the problem.

I express my appreciation to the National Academy of Sciences-National Research Council for granting me the opportunity to participate in their postdoctoral research program, and in particular to the National Bureau of Standards for its support of my research under this program. Also, I thank Mrs. Karen Bedeau, who wrote the code for computing tables 1,2 , and 3 .
TABLE 3. Percentage points $\mathrm{u}_{2}$ to be used in conjunction with table 1 for obtaining simultaneous upper and lower bounds on. the roots of a bivariate Wishart matrix

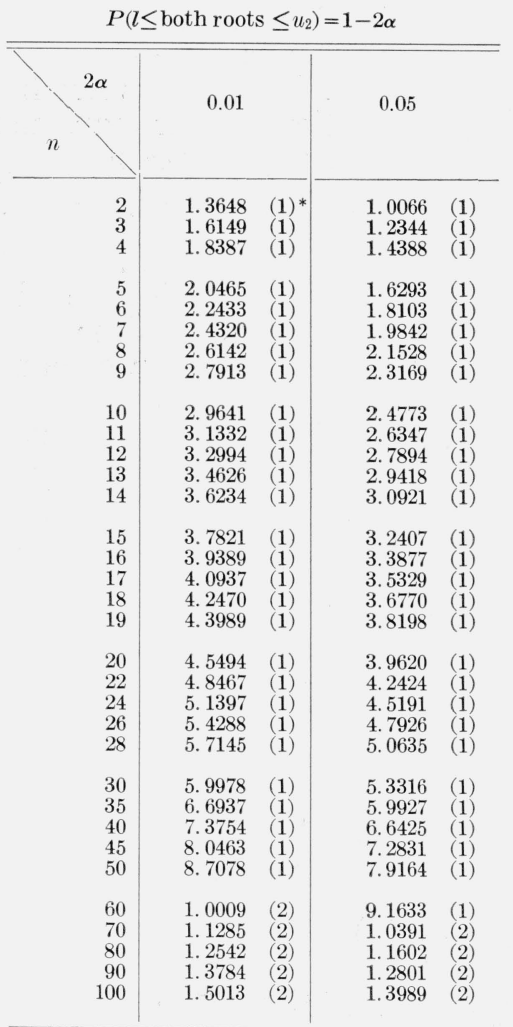

*The symbol (i) means that the tabled entry is to be multiplied by $10^{i}$.

\section{References}

[1] T. W. Anderson, An introduction to multivariate statistical analysis (John Wiley \& Sons, Inc., New York, N.Y., 1958).

[2] T. W. Anderson, J.R.S.S. series B, 10, 132-139 (1948).

[3] Edward Tankard Brown, Introduction to the theory of determinants and matrices (The University of North Carolina Press, Chapel Hill, 1958).

[4] R. Gnanadesikan, Contributions to multivariate analysis including univariate and multivariate variance components analysis and factor analysis, Institute of Statistics, University of North Carolina, Mimeo Series, No. 158 (1956).

[5] Frank E. Grubbs, On estimating precision of measuring instruments and product variability, J.A.S.A. 43, 243-264 (1948).

[6] D. L. Heck, Charts of some upper percentage points of the distribution of the largest characteristic root, Ann. Math. Stat. 31, 625-642 (1960).

[7] K. C. Sreedharan Pillai, Statistical tables for tests of multivariate hypotheses (The Statistical Center, University of the Philippines, Manila, 1960).

[8] S. N. Roy and R. C. Bose, Simultaneous confidence interval estimation, Ann. Math. Stat. 24, 513-536 (1953).

[9] S. N. Roy and R. Gnanadesikan, Further contributions to multivariate confidence bounds, Biometrika 44, 399-410 (1957).

[10] S. N. Roy, Some aspects of multivariate analysis (John Wiley \& Sons, Inc., 1957).

[11] W. A. Thompson, Jr., Precision of simultaneous measurement procedures (in preparation 1962). 